\title{
Association between Painful Physical Symptoms and Clinical Outcomes in Korean Patients with Major Depressive Disorder:A Three-Month Observational Study
}

\section{Min Soo Lee ${ }^{1}$ \\ Sun Young Yum ${ }^{2}$ \\ Jin Pyo Hong ${ }^{3}$ \\ Se Chang Yoon ${ }^{4}$ \\ Jai Sung $\mathrm{Noh}^{5}$ \\ Kwang Hun Lee ${ }^{6}$ \\ Jung Ki Kim ${ }^{7}$ \\ Sang Yeol $\mathrm{Lee}^{8}$ \\ Pritibha Singh ${ }^{9}$ \\ Tamas Treuer ${ }^{10}$ \\ Victoria Reed ${ }^{9}$ \\ Joel Raskin ${ }^{11}$}

'Department of Psychiatry,

Korea University

College of Medicine, Seoul,

2Eli Lilly \& Company, Seoul,

3Department of Psychiatry,

Asan Medical Center,

Ulsan University, Seoul,

${ }^{4}$ Department of Neuropsychiatry,

Samsung Medical Center,

Sungkyunkwan University, Seoul,

${ }^{5}$ Department of Psychiatry,

Ajou University

School of Medicine, Suwon,

'Department of Neuropsychiatry,

Kyoungju Medical Center,

Donguk University, Gyeongju,

Department of Neuropsychiatry,

Pusan Merinol Medical Center, Busan,

Department of Psychiatry, Wonkwang

University Hospital, Iksan, Korea

'Eli Lilly \& Company,

Intercontinental Information Sciences, Sydney, Australia

${ }^{10}$ Eli Lilly \& Company, Budapest, Hungary

${ }^{11}$ Lilly Research Laboratories,

Eli Lilly \& Company, Toronto, Canada

\section{Correspondence}

Sun Young Yum

Lilly Korea, 7th Fl. Shin An Bldg.

943-19 Daechi-dong, Gangnam-gu,

Seoul 135-280, Korea

Tel $+82-2-6230-5837$

Fax +82-2-3452-3868

E-mail yumsu@lilly.com

(c) This is an Open Access article distributed under the terms of the Creative Commons Attribution Non-Commercial License (http://creativecommons.org/licenses/by-nc/3.0) which permits unrestricted non-commercial use, distribution, and reproduction in any medium, provided the original work is properly cited.
Objective This paper aims to examine the association between painful physical symptoms (PPS) and major depressive disorder (MDD) in a naturalistic clinical practice setting within a Korean population.

Methods Patients with acute MDD that joined a multicountry, observational, three-month study in six Asian countries and regions were classified as PPS+ (mean score $\geq 2$ ) and PPS(mean score $<2$ ) using the modified Somatic Symptom Inventory. In this analysis, we report the results from the Korean subset, where depression severity was assessed using the Clinical Global Impression of Severity (CGI-S) scale and 17-item Hamilton Depression Rating Scale $\left(\mathrm{HAMD}_{17}\right)$. Pain severity was measured using a visual analogue scale (VAS), while the EuroQoL (EQ-5D) assessed patient well-being.

Results Of 198 patients, $45.96 \%$ (91/198) of patients were classified as PPS+, of which $78.02 \%(71 / 91)$ were women. PPS+ patients had significantly more severe depression at baseline \{CGI-S score, mean [standard deviation (SD)], PPS+: 5.09 [0.79]; PPS-: 4.63 [0.76]; $\mathrm{p}<0.001 ; \mathrm{HAMD}_{17}$ total score, mean [SD], PPS+: 24.34 [5.24]; PPS-: 20.76 [5.12]; $\mathrm{p}<0.001\}$ and poorer quality of life [EQ-5D overall health state, mean (SD), PPS+: 39.37 (20.52); PPS-: 51.27 [20.78]; $<<0.001]$ than PPS- patients. Both groups improved significantly $(\mathrm{p}<0.001)$ in depression and pain severity outcomes, as well as quality of life by endpoint, but no significant within-group baseline-to-endpoint change wase observed.

Conclusion The frequency of PPS was common in Korean patients with MDD, and was associated with more severe depression, poorer quality of life, and a trend towards poorer clinical outcome.

Psychiatry Invest 2009;6:255-263

Key Words Pain, Depressive disorder, Major, Korea, Quality of life, Depression, Culture.

Received: June 25, 2009 Revised: October 5, 2009 Accepted: October 27, 2009

Available online: December 8, 2009

\section{Introduction}

Major depressive disorder (MDD) currently affects an estimated 340 million people worldwide. ${ }^{1}$ In recent years, the point prevalence of MDD among Koreans has been rising in all age groups. ${ }^{2}$ A 2007 study reported the 12-month prevalence of MDD among Koreans to be $1.7 \%$ with a lifetime prevalence of $4.3 \%$, and roughly twice the rate in women. ${ }^{3}$

Researchers speculate that the incidence of new cases of depression is currently underestimated, ${ }^{7}$ where underlying psychological symptoms may be masked by the higher incidence of concomitant painful physical symptoms (PPS), especially in Asian cultures. In these cultures, patients tend to somatize instead of openly expressing their emotional suffering and then seek somatic treatment. ${ }^{8-11}$

Kirmayer ${ }^{12}$ stated that culture carries great influence on the expression of an individual's mental health or illness. Other researchers have also speculated that ethni- 
city may influence the somatic and psychiatric occurence of depression. ${ }^{13,14}$ For example, in Korean immigrant culture within the USA, when compared to other cultures, Koreans are less likely to seek medical attention for anxiety, depression or other psychological problems, ${ }^{15}$ most likely given their strong belief that depression is a normal part of life rather than a mental illness. ${ }^{11,16}$

Depression accompanied by painful physical symptoms is also not unfamiliar to Koreans. Although controversial, it has been suggested that a Korean folk syndrome termed "hwa-byung", is a culturally patterned way that some Koreans experience depression. ${ }^{17}$ Hwa-byung is thought to stem from suppressed emotions, manifesting as hostility, a sense of misery, and accompanied by somatic symptoms ${ }^{15,18-21}$ such as nonspecific gastrointestinal problems and generalized aches and pains. ${ }^{22} \mathrm{Re}-$ search from other cultures reports that PPS, including headaches, stomach pain, back pain and vague, poorly localized pain, are reported by up to $76 \%$ of all patients with depression. , $23-25^{-25}$

High prevalence of PPS in depressed patients has been established in American, ${ }^{24}$ European, ${ }^{26}$ and Latin Ameri$\mathrm{can}^{25}$ general and psychiatric practice settings, as well as Asian psychiatric settings. ${ }^{27}$ This paper examines PPS in Korean patients with MDD in psychiatric outpatient settings.

This analysis is part of a larger study covering 6 Asian countries/regions, with the primary objective of evaluating the frequency of PPS in Asian patients treated for an acute MDD episode in a naturalistic clinical practice setting. Here we report the frequency of PPS in the Korean subpopulation and provide clinical information from a Korean perspective, describing the changes in disease severity, treatment patterns, and quality of life observed during the 3-month observational period. We then compare participant outcomes based on the presence (PPS+) or absence (PPS-) of painful physical symptoms.

\section{Methods}

\section{Patients}

Female and male psychiatric inpatients or outpatients who were older than 18 years of age, with a primary diagnosis of MDD [Diagnostic and Statistical Manual of Mental Disorders, Fourth Edition Text Revision (DSMIV-TR $)^{28}$ or the International Classification of Diseases, Tenth Revision (ICD-10) ${ }^{29}$ ], Clinical Global Impressions of Severity (CGI-S) Scale $\geq 430$ and who agreed to receive antidepressant treatment, were enrolled at the discretion of their treating psychiatrist. Patients were enrolled from 7 medical centers including 6 university hospitals throughout Korea.
Patients were not eligible for enrollment if they had any previous or current diagnosis of schizophrenia, schizophreniform disorder, schizoaffective disorder, bipolar disorder or dementia. Patients were also excluded from the study if their current depressive episode had persisted for $\geq 6$ months, if they were experiencing treatment-resistant chronic pain or had a painful inflammatory condition related to an identifiable medical condition.

\section{Study design}

This prospective, non-interventional, epidemiological, three-month observational study was designed to assess the frequency of PPS occurrence among patients from 6 Asian countries/regions treated in naturalistic clinical settings for an acute episode of MDD. Patients were recruited from 14 June 2006 to 15 February 2007. We now present data from a subanalysis of all Korean patients who completed both baseline and endpoint visits.

Treatment for MDD was prescribed through the usual standard of care by the treating physician, and was not provided or directed by the study sponsor. All subsequent treatment changes were solely at the discretion of the physician and the patient, and there was no attempt by the sponsor to influence prescribing patterns.

Upon joining the study entry, patients were assessed for the presence of PPS as measured by their mean score on the pain related items of the modified Somatic Symptom Inventory (SSI)[items 27 (joint pain) and 28 (neck pain) were added]. ${ }^{31}$ Patients with a mean score of $\geq 2$ were defined as painful physical symptom positive (PPS + ) and the remaining patients were defined as painful physical symptom negative (PPS-). The SSI items are rated on a scale of 1 to $5 ; 1$ is defined as "not at all" and 2 to 5 indicate presence of symptoms in increasing severity. ${ }^{32}$

Following enrollment, patients were observed for three months. To collect data during the study, there was a total of two visits, at baseline and study end. Upon entering the study, patients were assessed for eligibility and consent to release information was received from each patient or their legal representative prior to enrolment.

This study was not designed to assess the overall safety profile of any study drug. The study investigators had an obligation to report any spontaneous treatmentemergent adverse events and serious adverse events, just as they would in normal clinical practice.

The study protocol (study code: F1J-AA-B015) was submitted to and subsequently approved by the local regulatory agency as well as each institutional review board. This study was carried out in accordance with the ethical principles that have their origin in the Declaration of Helsinki. These principles are also consistent with good clinical practices in addition to Korean laws and regulations. 


\section{Measures}

Demographic and clinical data, including disease history, work, hospitalization and medical comorbidities, were collected at baseline. Clinical data was also collected at endpoint. Depression severity was measured using the 17-item Hamilton Depression Rating Scale (HA$\left.\mathrm{MD}_{17}\right)^{33}$ and CGI-S. Response was defined as a $\geq 50 \%$ reduction in $\mathrm{HAMD}_{17}$ total score from baseline to endpoint. Remission was defined by a $\mathrm{HAMD}_{17}$ total score of $\leq 7$ at endpoint. Pain severity was measured using a Visual Analog Scale (VAS) ${ }^{34}$ and patient quality of life was assessed using the EuroQol Questionnaire-5 Dimensions (EQ-5D). The EQ-5D is a self-administered questionnaire that provides a utility score related to health state and impact on usual life activities including mobility, self-care, usual activities, pain/discomfort and anxiety/depression. A set of weights that represent the general population's preferences is employed to create the index. ${ }^{35}$ For the EQ-5D scale, a United Kingdom population was used to standardize scores as a Korean population has not been validated.

\section{Statistical analysis}

The primary analytical objective for this study, to estimate the frequency of PPS within the full study cohort, has been previously reported. ${ }^{27}$ Demographic characteristics and treatment patterns of patients were evaluated across PPS groups using: 1) two-sample t-test or nonparametric Wilcoxon rank sum test for continuous variables, 2) Fisher's exact test for categorical variables and 3) analysis of covariance (ANCOVA) for changes from baseline on clinical outcome variables HAMD $_{17}, \mathrm{CGI}-\mathrm{S}$, VAS and EQ-5D) adjusted for propensity scores. In this study the propensity score represents the conditional probability of a patient being PPS + given their baseline data. The propensity score was calculated with a logistic regression model using the following baseline variables defined a priori: gender, age, age at first episode, illness severity, previous depressive episodes (yes/no), number of previous depressive episodes, number of significant pre-existing comorbidities and previous depression treatment (drug and non-drug). In this study, the propensity score represents the conditional probability of a patient being PPS+ given their baseline data. Further interaction terms determined post hoc included CGI-S with EQ-5D, SSI with VAS and EQ-5D with number of significant preexisting comorbidities. ${ }^{36}$

Comparisons between selective serotonin reuptake inhibitor (SSRI) and serotonin and norepinephrine reuptake inhibitor (SNRI) monotherapy groups were evaluated at baseline and for changes from baseline (adjusted for baseline, age and sex). Logistic regression was used to evaluate the number of patients achieving response and remission, with comparisons made between PPS groups (adjusted for propensity scores). All statistical analyses were performed using $\mathrm{SAS}^{\circledR}$ Version 8.2 for Windows $^{\text {TM }}$ (SAS Institute, Cary, NC). No adjustment for multiple comparisons was performed. The level of statistical significance was defined a priori as alpha $=0.05$.

\section{Results}

\section{Patient disposition}

A total of 198 patients were enrolled in Korea, of which 46.0\% (91/198) were PPS+ and 54.0\% (107/198) were PPS-. The overall completion rate in the study was $74.2 \%(147 / 198)$, with $71.4 \%(65 / 91)$ of completers in the PPS+ group and $76.6 \%(82 / 107)$ of completers in the PPS- group. Forty-eight patients were lost to followup [28.6\% (26/91) PPS+ patients and 20.6\% (22/107) PPS- patients]. In the PPS- group, two patients voluntarily discontinued and one patient committed suicide during the study. There were no statistically significant between-group differences regarding reasons for discontinuation.

\section{Demographic and disease characteristics at baseline}

The demographic and disease characteristics of PPS+ and PPS- patients at baseline are detailed in Table 1 . Significantly more patients in both groups were women [overall: $68.7 \%(136 / 198), \mathrm{p}=0.009$ ]. Patients had a mean [standard deviation (SD)] age of 49.5 (14.17) years and a mean body mass index (BMI)(SD) of $22.9(3.05) \mathrm{kg} / \mathrm{m}^{2}$. Overall, patients experienced their first depressive episode at the mean age (SD) of 46.5 (14.1) years, 35.9\% $(66 / 184)$ of patients had one or more previous depressive episodes with a mean (SD) episode duration of 11.4 (10.14) weeks. In the PPS+ group, the mean (SD) duration of the current depressive episode was 8.7 (7.83) weeks, while in the PPS- group it was 7.6 (5.84) weeks, but not statistically significant. The most frequently reported medical comorbidities were cardiovascular disease [PPS+: 15.4\% (14/91); PPS-: 16.8\% (18/107)], diabetes mellitus [PPS+: 8.8\% (8/91); PPS-: 6.5\% (7/107)] and gastrointestinal disease [PPS+: 7.7\% (7/91); PPS-: 7.5\% $(8 / 107)]$.

\section{Depression measures}

At baseline, PPS+ was associated with significantly greater severity of depression and poorer clinical status than PPS- based on CGI-Severity score [PPS+ Mean (SD): 5.1 (0.79); PPS-: 4.6 (0.76); p<0.001], HAMD 17 total score [PPS+ Mean (SD): 24.3 (5.24): PPS-: 20.8 
TABLE 1. Patient demographics and disease severity at baseline

\begin{tabular}{lcccc}
\hline & PPS+ $(\mathrm{n}=91)$ & PPS- $(\mathrm{n}=107)$ & Overall (N=198) & $\mathrm{p}$-value \\
\hline Age, mean (SD) & $50.0(14.77)$ & $49.2(13.70)$ & $49.5(14.17)$ & $0.712^{*}$ \\
Female n, (\%) & $71(78.0)$ & $65(60.8)$ & $136(68.7)$ & $0.009^{* * *}$ \\
Body Mass Index (kg/m²), mean (SD) & $22.9(2.68)$ & $22.9(3.33)$ & $22.9(3.05)$ & $0.999^{*}$ \\
Age at first episode of MDD, mean (SD) & $46.7(14.24)$ & $46.4(14.13)$ & $46.5(14.14)$ & $0.900^{*}$ \\
Number of previous episodes, median (range) & $2.0(1.0-18.0)$ & $2.0(1.0-10.0)$ & $2.0(1.0-18.0)$ & $0.989^{* *}$ \\
$\geq 1$ previous episode, $\mathrm{n}(\%)$ & $30(38.0)$ & $36(34.3)$ & $66(35.9)$ & $0.643^{* * *}$ \\
Duration of current episode, weeks, median (range) & $6.0(2.00-53.00)$ & $6.0(1.00-32.00)$ & $6.0(1.00-53.00)$ & $0.425^{* *}$ \\
Last episode, weeks, median (range) & $12.0(1.00-48.00)$ & $8.0(2.00-52.00)$ & $8.0(1.00-52.00)$ & $0.030^{* *}$ \\
Time between remission of last and start of current & $24.0(9.00-192.00)$ & $24.0(8.00-670.00)$ & $24.0(8.00-670.00)$ & $0.716^{* *}$ \\
episode, weeks, median (range) & & & & \\
CGl-severity, mean (SD) & $5.1(0.79)$ & $4.6(0.76)$ & $4.8(0.81)$ & $<0.001^{*}$ \\
HAMD 17 total score, mean (SD) & $24.3(5.24)$ & $20.8(5.12)$ & $22.3(5.46)$ & $<0.001^{*}$ \\
HAMD 17 Anxiety/Somatization, mean (SD) & $8.7(2.44)$ & $7.5(2.90)$ & $8.0(2.77)$ & $0.006^{*}$ \\
HAMD 17 Core mood, mean (SD) & $8.7(2.96)$ & $7.5(2.45)$ & $8.0(2.74)$ & $0.007^{*}$ \\
EQ-5D health state score, median (range) & $40.0(0-85.00)$ & $50.0(0-99.00)$ & $50.0(0-99.00)$ & $0.001^{* *}$ \\
VAS, median (range) & & & & \\
Overall pain & $62.0(7.0-100.00)$ & $39.0(0-100.00)$ & $50.0(0-100.00)$ & $<0.001^{* *}$ \\
Headache & $50.0(0-100.00)$ & $11.0(0-93.00)$ & $30.0(0-100.00)$ & $<0.001^{* *}$ \\
Back pain & $38.0(0-100.00)$ & $4.0(0-100.00)$ & $10.0(0-100.00)$ & $<0.001^{* *}$ \\
Shoulder pain & $40.0(0-100.00)$ & $5.0(0-100.00)$ & $10.0(0-100.00)$ & $<0.001^{* *}$ \\
Pain interference & $67.0(0-100.00)$ & $31.5(0-100.00)$ & $50.0(0-100.00)$ & $<0.001^{* *}$ \\
Pain while awake & $62.0(0-100.0)$ & $20.0(0-100.0)$ & $45.0(0-100.0)$ & $<0.001^{* *}$ \\
\hline
\end{tabular}

${ }^{*} \mathrm{p}$-value using t-test for comparison of means, ${ }^{* *} \mathrm{p}$-value using non-parametric Wilcoxon Rank Sum Test, ${ }^{* * *} \mathrm{p}$-value using Fisher's Exact Test. PPS+: painful physical symptom positive group, PPS-: remainder of study population, n: number of patients in each group, N: number of patients in the total study population, SD: standard deviation, MDD: major depressive disorder, CGI-Severity: Clinical Global Impressions of Severity Scale, HAMD17: 17-item Hamilton Depression Rating Scale, VAS: Visual Analog Scale, EQ-5D: EuroQol Questionnaire-5 Dimensions

(5.12); $<<0.001]$, HAMD anxiety/somatization [PPS+ Mean (SD): 8.7 (2.44); PPS-: 7.5 (2.90); $\mathrm{p}=0.006]$ and HAMD core mood subscores [PPS+ Mean (SD): 8.7 (2.96); PPS-: 7.5 (2.45); $\mathrm{p}=0.007]$ (Table 1). From baseline to endpoint, there was a statistically significant $(\mathrm{p}<$ 0.001 ) improvement in all depression severity measures in both PPS + and PPS- patients (adjusted mean change from baseline to endpoint) (Figures 1 and 2); no significant between-group differences were found. There were no statistically significant differences between groups in terms of the proportion of responders or remitters at endpoint [difference in response (PPS+ minus PPS-): -0.06 , $95 \%$ CI $(-0.21,0.10), \mathrm{p}=0.487$; difference in remission (PPS+ minus PPS-): $-0.06,95 \%$ CI $(-0.22,0.10), \mathrm{p}=$ 0.506].

\section{Pain measures}

As expected, PPS + patients reported significantly greater $(p<0.001)$ pain severity at baseline than PPS- patients based on the VAS overall and subscales (Table 1). At endpoint, patients in both groups experienced a significant improvement in pain severity [adjusted mean change from baseline; $\mathrm{p}<0.001$ for PPS + and PPS- patients on VAS overall and all subscales, except for PPS+

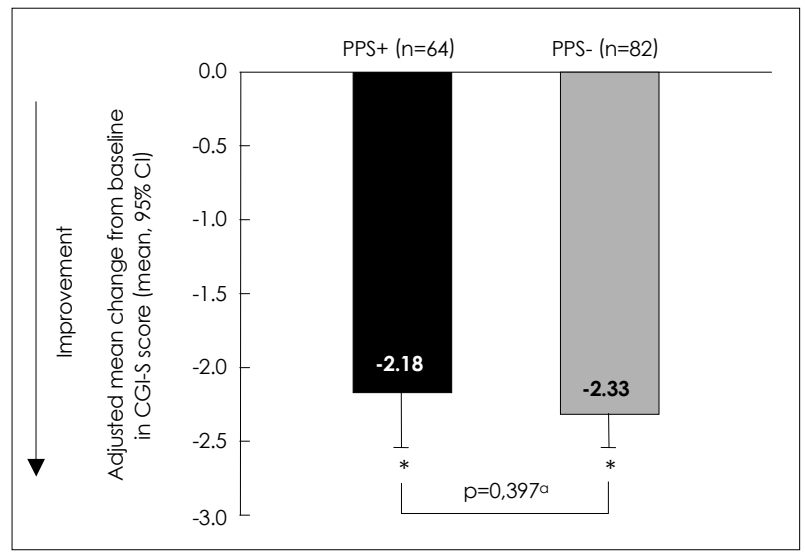

FIGURE 1. Adjusted mean change from baseline to endpoint in CGI-S total score. ${ }^{a} \mathrm{p}$-value for adjusted mean difference [0.15, $95 \% \mathrm{Cl}(-0.21,0.51)]$ using Type III Sums of Squares from analysis of covariance (ANCOVA) model: change=PPS group, propensity score and baseline. *Within group $p$-value $(p<0.001)$ from ANCOVA model: change=PPS group, propensity score and baseline. PPS+: painful physical symptom positive group, PPS-: remainder of study population, CGI-S: Clinical Global Impressions of Severity Scale, $\mathrm{n}$ : number of patients in each group, $\mathrm{Cl}$ : confidence interval.

patients with shoulder pain $(\mathrm{p}=0.008)]$, improvement being prominent in the pain interference, pain while awake, and headache subscales (Figure 3). Between-group dif- 
ferences in pain severity measures from baseline to endpoint were not statistically significant (Figure 3).

\section{Quality of life measures}

At baseline, PPS + patients had significantly $(\mathrm{p}<0.001)$ lower quality of life [EQ-5D health state score mean (SD): 39.4 (20.52); EQ-5D utility score mean (SD): 0.2 (0.36)] than PPS- patients [EQ-5D health state score mean (SD): 51.3 (20.78); EQ-5D utility score mean (SD): 0.5 (0.30)]. Both groups of patients had statistically signifi-

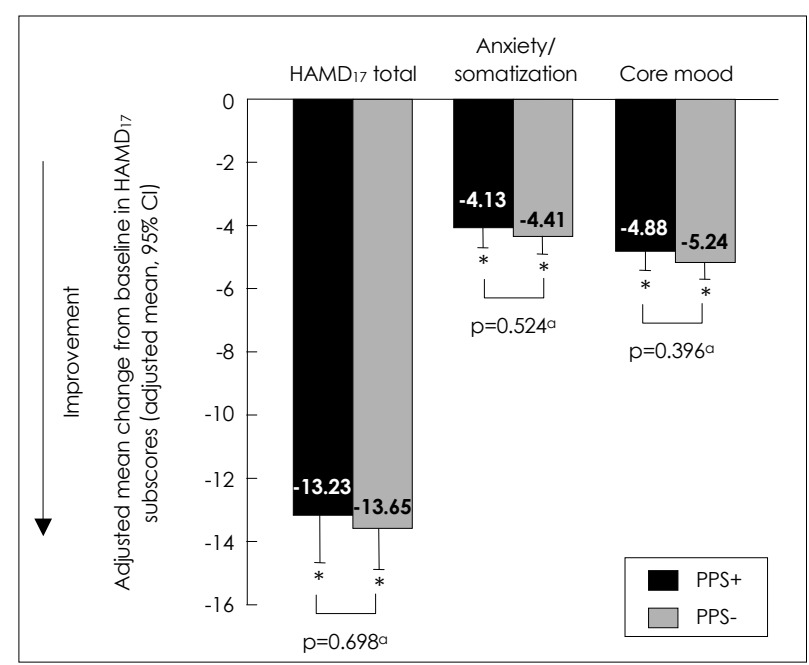

FIGURE 2. Adjusted mean change from baseline to endpoint in $\mathrm{HAMD}_{17}$ total score, anxiety/somatization and core mood. ${ }^{\mathrm{a}} \mathrm{p}$-values for adjusted mean differences $\left[\mathrm{HAMD}_{17}\right.$ total score $=0.41$, $95 \% \mathrm{Cl}(-1.69,2.51)$; Anxiety/somatization subscore $=0.28,95 \%$ $\mathrm{Cl}(-0.59,1.16)$; Core mood subscore $=0.36,95 \% \mathrm{Cl}(-0.47,1.19)]$ using Type III Sums Of Squares from analysis of covariance (ANCOVA) model: change=PPS group, propensity score and baseline. *Within group $p$-value $(p<0.001)$ from ANCOVA model: change=PPS group, propensity score and baseline, and is the same for $\mathrm{HAMD}_{17}$ total score and both subscores that are presented here. PPS+: painful physical symptom positive group, PPS-: remainder of study population, $\mathrm{HAMD}_{17}$ : 17 -item Hamilton Depression Rating Scale, Cl: confidence interval. cant improvements in quality of life $(\mathrm{p}<0.001)$ from baseline to endpoint PPS+ [adjusted mean change in EQ5D health state score (95\% CI)]: 20.9 [16.11, 25.64]; adjusted mean change in EQ-5D utility score [95\% CI]: $0.3[0.27,0.40]$ \} \{PPS- [adjusted mean change in EQ$5 \mathrm{D}$ health state score $(95 \% \mathrm{CI})]: 24.0$ [19.78, 28.22]; adjusted mean change in EQ-5D utility score [95\% CI]: $0.4[0.36,0.47]\}$.

\section{Work and hospitalization}

During the three months prior to the study, 74.4\% (67/ 90) of PPS+ patients and 65.1\% (69/106) of PPS- patients were unemployed. During the three-month study period, $68.5 \%(50 / 73)$ of PPS+ patients and 67.0\% (61/91) of PPS- patients were unemployed. Patients that did work during the study period worked on average a total of 40 hours per week and did not miss a day of work due to MDD (range 0-9 days).

A total of eight patients required psychiatric hospitalization during the study. In the PPS+ group, $7.8 \%$ of the patients were hospitalized, whereas $3.8 \%$ of the patients in the PPS- group were hospitalized; however, these results were not statistically significant. During the study, $4.7 \%$ of PPS+ patients and $1.3 \%$ of PPS- patients required 29 days or more of hospitalization.

\section{Treatment patterns}

Of 197 patients reporting treatment at study entry, 53 patients were not receiving any treatment and 144 patients were receiving one or more treatments. There was no statistically significant difference between PPS + or PPS- patients in terms of medication class, number, and psychosocial therapy. A small proportion of patients [11.4\% (22/193)] were receiving some treatment for pain at baseline. More PPS+ patients [12.2\% (11/90)] used one or more classes of concomitant treatment.
FIGURE 3. Adjusted mean change from baseline to endpoint in VAS overall and subscales. ${ }^{a} p$-values for adjusted mean difference using Type III Sums Of Squares from analysis of covariance (ANCOVA) model: change=PPS group, propensity score and baseline. *Within group p-value from ANCOVA model: change=PPS group, propensity score and baseline. PPS+: painful physical symptom positive group, PPS-: remainder of study population, VAS: Visual Analog Scale, $\mathrm{Cl}$ : confidence interval.

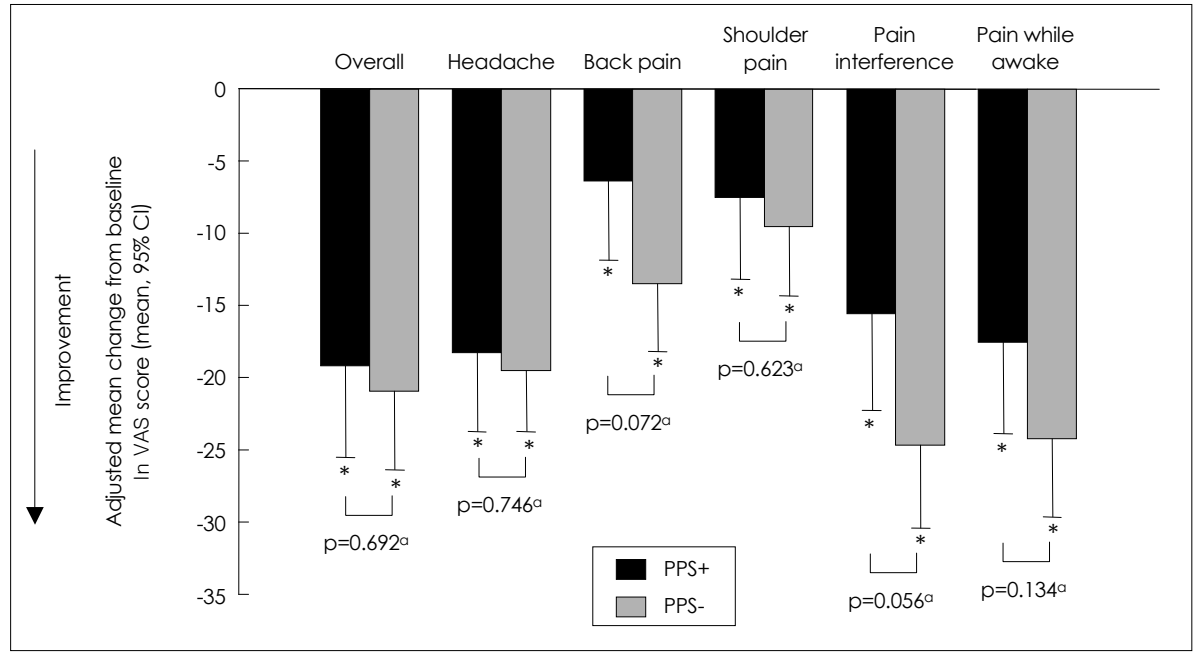


The most commonly prescribed antidepressants at baseline and at endpoint were the SSRI paroxetine and escitalopram, followed by mirtazapine and venlafaxine. The most common reason for discontinuation of MDD treatment was inadequate response to treatment, followed by the investigator's or patient's perception of cure. A total of eight patients discontinued their MDD medication due to an adverse event

There was no statistically significant difference between treatments with SSRI [at baseline: PPS $+\mathrm{n}=39$ (42.9\%); PPS- $n=52(48.6 \%)$ ] versus SNRI [at baseline: PPS $+n=15$ (16.5\%); PPS- $n=9$ (8.4\%)] monotherapy from baseline to endpoint. This was the case in all enrolled patients in terms of depression severity and quality of life as measured by CGI-S, HAMD 17 total score, $\mathrm{HAMD}_{17}$ anxiety/somatization, HAMD ${ }_{17}$ core mood and EQ-5D health state and utility scores. However, both groups of patients did experience statistically significant improvement in depression severity and quality of life with both treatments. Similar results were obtained when comparing both treatments based on the VAS as a pain severity measure. Overall, statistically significant pain relief was experienced by patients receiving both SSRI and SNRI treatments regarding overall pain, headache severity, pain interference and pain while awake.

\section{Discussion}

In this Korean subanalysis of a multicountry, threemonth, prospective, noninterventional study in Asian patients with MDD, ${ }^{27}$ the frequency of PPS in the Korean subpopulation was found to be $46.0 \%$. Although clinicians may assume that as a result of greater cultural acceptance, PPS are more frequent in Asians, ${ }^{37,38}$ the observations in this study do not support this assumption. The frequency of PPS reported in Koreans was comparable to the prevalence found in Europe $(43.0 \%){ }^{26}$ but lower than that reported in the overall Asian study $(51.8 \%),{ }^{27}$ and even lower when compared with results reported in North America $(69.0 \%)^{24}$ and Latin America $(73.0 \%)^{25}$

PPS reporting is usually linked to reporting and interpretational bias, can be culturally sensitive and also reflects country-specific training practices of health care professionals, for instance, how complaints are explored Country-specific data and subanalyses can, therefore, help to reveal such country-specific differences, while cross cultural studies can indicate potential linkages between cultural values, socialization practices and symptom reporting. ${ }^{39,40}$ Additionally, the study was conducted in university hospitals in Korea, which exist as tertiary referral centers. As a result, patients treated in major university hospitals cannot be said to be representative of the MDD population in Korea. Although this study was carried out in patients presented to psychiatrists in the cultural ambience of Korea, patients with MDD and PPS may be more likely to present to other specialties with complaints of pain as opposed to psychiatrists with depression.

In Korean patients with acute MDD, the presence of PPS at baseline was associated with a greater severity of depression and poorer quality of life. Similar outcomes were observed for the overall study and other evaluated Asian subpopulations evaluated. ${ }^{27}$ By study end, both PPS+ and PPS- patients had improved significantly in terms of their depression and quality of life. There was a trend towards more severe depression and poorer quality of life for PPS + patients compared to the overall study sample. However, no statistically significant difference in terms of depression and quality of life between PPS+ and PPS- patients was observed by study end.

As the Korean patient sample was predominantly comprised of women who had experienced their first depressive episode later in life (46.5 years of age on average) than commonly expected, ${ }^{41}$ it is possible that these patients were going through perimenopausal or menopausal depression, which is commonly related to PPS. ${ }^{42}$ Also of note was the high rate of unemployment observed in both PPS + and PPS- patients in the three months prior to the study commencement and during the study period in this Korean sample. However, the assessment did not differentiate between unemployment and women working full time as housewives, who were also classified as unemployed. Following the financial crisis in 1997, Korean workers no longer enjoyed lifetime employment that had previously characterized the work environment in this country. ${ }^{43}$ Instead, Korean companies have enforced early retirement, honorary retirement and layoffs to reduce their payrolls. ${ }^{43}$ In 2003, Jang reported that only $60.0 \%$ of Korean workers in their early thirties will still be employed by the time they become 50 years old. $^{44}$

In terms of treatment, a previous study has verified the utility of SNRIs (duloxetine) as efficacious and safe treatments for emotional and physical symptoms of MDD in a predominantly Asian patient sample. ${ }^{45}$ In the Korean subpopulation, no statistically significant betweengroup difference in treatments were found with SSRIs and SNRIs. While there were no statistical differences between PPS+ and PPS-, the PPS+ group did show a trend to fare less well in their treatment. The PPS+ group experienced lower completion rates, higher hospitalization rates with more stays over 29 days, more required classes of treatment, and less improvement in all efficacy 
measures. Antidepressant selection patterns based on psychiatric symptoms with and without PPS were not considered in the statistical comparison, but might be undertaken in further analyses.

Depressive symptoms in Korean people can be perceived in a complex way, intricately overlapping in all domains of a person's existence. ${ }^{46}$ DSM-IV currently lists hwa-byung as a Korean culture-bound syndrome typically associated with middle-aged and elderly women that results from distressed emotions and passive suffering. It is related specifically to the Korean way of perceiving and reacting to intolerable situations. ${ }^{15}$ Furthermore, it is characterized by predominantly physical symptoms of depression and anhedonia, generally lacking subjective awareness of depressed feelings. This is comparable to Kohut's concept, where affective disorders result from extreme fear, threat to self-esteem or worth and the shame of being faced with failure (narcissistic rage). ${ }^{47}$ Similarly, McDougall associated the presence of somatic symptoms in alexithymic patients with a response or defense mechanism against frightening experiences. ${ }^{48}$

Just as depression has a complex biopsychosocial etiopathogenesis, perception of pain is also a complex phenomenon. The subjective experience of pain results from central integration of painful stimuli, along with emotional and cognitive factors. It is suggested that depression may contribute to increased excitability of the central nociceptive pathways via the limbic system input, ${ }^{49,50}$ and ultimately contribute to pain sensation. Chronic pain may also contribute to depression. In chronic pain, the somatosensory pathway is not activated, but rather the depression pathway, with signals from the limbic system. The kindling model has been applied to pain-induced neuroplastic changes and corticolimbic sensitization, leading to a clinical picture of persistent pain, affective dysregulation and disturbances in behavior. ${ }^{51}$

Along with the stigma of mental illness, Koreans in general tend to have a greater acceptance of physical rather than mental pain. In this context, it can be speculated that the frequency of PPS may be higher in Korean patients with MDD; however, this study cannot confirm or refute this hypothesis.

Results from the overall multicountry study revealed that PPS are experienced by approximately half of patients with MDD in Asia and are associated with poor clinical status and less pain reduction. ${ }^{27}$ The greater adjusted pain reduction experienced by PPS- patients may indicate that depression with substantial pain requires a different treatment strategy. This strategy would differ from SSRI treatment, which is what the majority of patients received. On the other hand, another possible explanation for less pain reduction in the presence of so- matic symptoms is Freud's concept of guilt, which sustains that self-flagellation, reflected in this case as the presence of somatic symptoms, is an expression of the patient's resistance to recovery. ${ }^{45}$

\section{Limitations}

It is important to note that the overall study of 6 Asian countries/regions may not be powered for country-specific analyses, and therefore, data should be interpreted with caution. Patients without postbaseline visits and measures for all covariates were excluded from the analysis, resulting in a reduced size of the country-specific analysis population. The findings presented for this Korean subpopulation are for descriptive and explorative purposes and must be interpreted within these confines.

Even though the gender findings were consistent with that of the overall study across all Asian cohorts $(68.7 \%$ to $78.0 \%$ of patients were women who presented PPS with an overall onset of first depressive episode at 41.0 years of age), ${ }^{27}$ these elements could be potential confounders. The high unemployment rates observed in this Korean population may be more related to the Korean employment pattern or the higher percentage of unemployed housewives, than the severity of their depression. This could be another confounder. Additionally, the validity of the pain scores used may not be well established and suitably devised for all ages and genders.

\section{Conclusions}

In this study, almost half of the Korean MDD patients experienced PPS. As PPS has been associated with more severe depression, lower quality of life and a trend towards poorer outcomes, clinical management should aim to address associated mental and physical symptoms. Our results support previous findings in Asia, indicating that the presence of PPS is associated with more severe depression. ${ }^{27}$ Overall, despite the established appreciation of pain perception complexities, further research is needed to explore the impact of such complexities on choices of effective treatment modalities.

\section{- Acknowledgments}

This study was sponsored by Eli Lilly and Company. The authors would like to thank Alan Brnabic (Eli Lilly and Company employee) for the design of the initial study and acknowledge the writing assistance provided by Keyra D. Martinez, MD, of Primo Scientific, Inc., Panama City, Panama.

The authors also thank Ms. Hee Jeong Kim, who managed the operations of this trial in Korea as well as all the sub-investigators and site coordinators.

Sun Young Yum, Pritibha Singh, Tamas Treuer, Victoria Reed and Joel Raskin are employees of Eli Lilly and Company. Min Soo Lee, Jin Pyo Hong, Se Chang Yoon, Jai Sung Noh, Kwang Hun Lee, Jung Ki Kim and Sang Yeol Lee are clinical investigators for the F1J-AA- 
B015 study (upon which the manuscript is based), for which their institutions were funded by Eli Lilly and Company for the time spent and assessment cost.

Joel Raskin holds shares in Eli Lilly. Se Chang Yoon has provided consultancy/advisory board work for Eli Lilly. Jai Sung Noh has received investigator initiated grants from Eli Lilly, Jansen-Cilag and Lundbeck. Jung Ki Kim has received investigator initiated grants from Eli Lilly and Sanofi-Aventis. Min Soo Lee has received investigator initiated grants from Eli Lilly, Wyeth, Sanofi-Aventis and Jansen-Cilag. Jin Pyo Hong has received investigator initiated grants from Sanofi-Aventis, Servie and Lundbeck and has provided consultancy/ advisory board work for Eli Lilly and GlaxoSmithKline. Kwang Hun Lee has provided consultancy/advisory board work for Jeil.

\section{REFERENCES}

1. Greden JF. Physical symptoms of depression: unmet needs. J Clin Psychiatry 2003;64:5-11.

2. Ohayon MM, Hong SC. Prevalence of major depressive disorder in the general population of South Korea. J Psychiatr Res 2006;40:30-36

3. Cho MJ, Kim JK, Jeon HJ, Suh T, Chung IW, Hong JP, et al. Lifetime and 12-month prevalence of DSM-IV psychiatric disorders among Korean adults. J Nerv Ment Dis 2007;195:203-210.

4. Kessler RC, Berglund P, Demler O, Jin R, Koretz D, Merikangas KR, et al. National Comorbidity Survey Replication. The epidemiology of major depressive disorder: results from the National Comorbidity Survey Replication (NCS-R). JAMA 2003;289:3095-3105.

5. Weissman MM, Bland RC, Canino GJ, Faravelli C, Greenwald S, Hwu $\mathrm{HG}$, et al. Cross-national epidemiology of major depression and bipolar disorder. JAMA 1996;276:293-299.

6. U.S. Census Bureau. Population Estimates by Demographic Characteristics, 2005.

7. Kirmayer LJ, Robbins JM, Dworkind M, Yaffe MJ. Somatization and the recognition of depression and anxiety in primary care. Am J Psychiatry 1993;150:734-741.

8. Rhi BY. Korean Ethos. Seoul: Korean Cultural Research Center, 1983.

9. Kim MT. Cultural influences of depression in Korean Americans. J Psychosoc Nurs Ment Health Serv 1995;33:13-18.

10. Pang KYC. Korean-American Women: Stories of acculturation and changing selves. New York: Routledge, 2006.

11. Bernstein KS, Lee JS, Park SY, Jyoung JP. Symptom manifestations and expressions among Korean immigrant women suffering with depression. J Adv Nurs 2008;61:393-402.

12. Kirmayer LJ. Cultural variations in the response to psychiatric disorders and emotional distress. Soc Sci Med 1989;29:327-339.

13. Berganza CE, Mezzich JE, Otero-Ojeda AA, Jorge MR, VillasenorBayardo SJ, Rojas-Malpica C. The Latin American guide for psychiatric diagnosis. A cultural overview. Psychiatr Clin North Am 2001;24: 433-446.

14. Parker G, Cheah YC, Roy K. Do the Chinese somatize depression? A cross-cultural study. Soc Psychiatry Psychiatr Epidemiol 2001;36: 287-293.

15. Pang KY. Hwabyung: the construction of Korean popular illness among Korean elderly immigrant women in the United States. Cult Med Psychiatry 1990;14:495-512.

16. Pang KYC. Symptoms of depression in elderly Korean immigrants: narration and the healing process. Cult Med Psychiatry 1998;22:93122.

17. Kim KI, Rhi BY. A review of Korean cultural Psychiatry. Transcultural Psychiatric Research Review 1976;13:101-114.

18. Lee SH. A study of the "Hwabyung" [Anger Syndrome]. Journal of Korea General Hospital 1977;1:63-69.

19. Park H, Murtgatroyd W, Raynock DC, Spillet MA. Relationship between intrinsic-extrinsic religious orientation and depressive symptoms in Korean-Americans. Counseling Psychology Quarterly 1998;11:
315-324.

20. Kim S, Rew L. Ethnic identity, role integration, quality of life, and depression in Korean-American women. Arch Psychiatr Nurs 1994; 8:348-356.

21. Lin KM, Lau JK, Yamamoto J, Zheng YP, Kim HS, Cho KH, et al. Hwa-byung. A community study of Korean Americans. J Nerv Ment Dis 1992;180:386-391.

22. Lin KM. Hwa-Byung: A Korean culture-bound syndrome? Am J Psychiatry 1983;140:105-107.

23. Corruble E, Guelfi JD. Pain complaints in depressed inpatients. Psychopathology 2000;33:307-309.

24. Bair MJ, Robinson RL, Eckert GJ, Stang PE, Croghan TW, Kroenke $\mathrm{K}$. Impact of pain on depression treatment response in primary care. Psychosom Med 2004;66:17-22.

25. Munoz RA, McBride ME, Brnabic AJ, Lopez CJ, Hetem LA, Secin R, Duenas HJ. Major depressive disorder in Latin America: the relationship between depression severity, painful somatic symptoms, and quality of life. J Affect Disord 2005;86:93-98.

26. Ohayon MM, Schatzberg AF. Using chronic pain to predict depressive morbidity in the general population. Arch Gen Psychiatry 2003;60:3947.

27. Lee P, Zhang M, Hong JP, Chua HC, Chen KP, Tang SW, et al. Frequency of painful physical symptoms with major depressive disorder in asia: relationship with disease severity and quality of life. J Clin Psychiatry 2009;70:83-91.

28. American Psychiatric Association. Diagnostic and Statistical Manual of Mental Disorders: Text Revision (DSM-IV-TR). 2000. Washington, DC: American Psychiatric Association.

29. World Health Organization [WHO]. International Classification of Diseases and related world health problems. 2007. Geneva, WHO.

30. Guy W. ECDEU assessment manual for psychopharmacology. Rockville, MD: US Department of Health and Human Service, Alcohol Drug Abuse and Mental Health Administration, NIMH Psychopharmacology Research Branch, 1976.

31. Kroenke K, Spitzer RL, Williams JB, Linzer M, Hahn SR, deGruy FV 3rd, et al. Physical symptoms in primary care. Predictors of psychiatric disorders and functional impairment. Arch Fam Med 1994;3:774779 .

32. Goldstein DJ, Lu Y, Detke MJ, Hudson J, Iyengar S, Demitrack MA. Effects of duloxetine on painful physical symptoms associated with depression. Psychosomatics 2004;45:17-28.

33. Hamilton M. A rating scale for depression. J Neurol Neurosurg Psychiatry 1960;23:56-62.

34. DeLoach LJ, Higgins MS, Caplan AB, Stiff JL. The visual analog scale in the immediate postoperative period: intrasubject variability and correlation with a numeric scale. Anesth Analg 1998;86:102-106.

35. EuroQol--a new facility for the measurement of health-related quality of life. The EuroQol Group 1990;16:199-208

36. Newgard CD, Hedges JR, Arthur M, Mullins RJ. Advanced statistics: the propensity score--a method for estimating treatment effect in observational research. Acad Emerg Med 2004;11:953-961.

37. Kleinman A. Neurasthenia and depression: a study of somatization and culture in China. Cult Med Psychiatry 1982;6:117-190.

38. Simon GE, VonKorff M, Piccinelli M, Fullerton C, Ormel J. An international study of the relation between somatic symptoms and depression. N Engl J Med 1999;341:1329-1335.

39. Varela RE, Vernberg EM, Sanchez-Sosa JJ, Riveros A, Mitchell M, Mashunkashey J. Anxiety reporting and culturally associated interpretation biases and cognitive schemas: a comparison of Mexican, Mexican American, and European American families. J Clin Child Adolesc Psychol 2004;33:237-247.

40. Pennebaker JW. Psychological factors influencing the reporting of physical symptoms. In: Stone AA, Turkkan JS, Bachrach CA, Jobe JB, Kurtzman HS, Cain VS, editors. The science of self-report: Implications for research and practice. New Jersey: Erlbaum Publishers, 1999, 
p.299-316

41. Zisook S, Lesser I, Stewart JW, Wisniewski SR, Balasubramani GK, Fava M, et al. Effect of age at onset on the course of major depressive disorder. Am J Psychiatry 2007;164:1539-1546.

42. Freeman EW, Sammel MD, Lin H, Nelson DB. Associations of hormones and menopausal status with depressed mood in women with no history of depression. Arch Gen Psychiatry 2006;63:375-382.

43. Park GS, Kim AE. Changes in attitude toward work and workers' identity in Korea. Korea Journal 2005;45:36-57.

44. Jang, JY. "Urinara goryeongja nodong sijang hyeonhwang" (The Labor Market for the Aging Workers of Korea). Research Paper. Seoul 2005: Korea Labor Institute.

45. Lee P, Shu L, Xu X, Wang CY, Lee MS, Liu CY, et al. Once-daily duloxetine $60 \mathrm{mg}$ in the treatment of major depressive disorder: multicenter, double-blind, randomized, paroxetine-controlled, non-inferiority trial in China, Korea, Taiwan and Brazil. Psychiatry Clin Neurosci 2007;61:295-307.
46. Park SY, Berstein KS. Depression and Korean American immigrants. Arch Psychiatr Nurs 2008;22:12-19.

47. Kohut H. The Analysis of the Self. New York: International Universities Press; 1971.

48. McDougall J. Theaters Of The Body: A Psychoanalytic Approach to Psychosomatic Illness. New York: W.W. Norton; 1989.

49. Janke EA, Holroyd KA, Romanek K. Depression increases onset of tension-type headache following laboratory stress. Pain 2004;111: 230-238.

50. Lipchik GL, Holroyd KA, O’Donnell FJ, Cordingley GE, Waller S, Labus $\mathrm{J}$, et al. Exteroceptive suppression periods and pericranial muscle tenderness in chronic tension-type headache: effects of psychopathology, chronicity and disability. Cephalalgia 2000;20:638-646.

51. Rome HP Jr, Rome JD. Limbically augmented pain syndrome (LAPS): kindling, corticolimbic sensitization, and the convergence of affective and sensory symptoms in chronic pain disorders. Pain Med $2000 ; 1: 7-23$ 